\title{
Erratum
}

In the article "Fasting Plasma Glucose and Incidence of Diabetes --- Implication for the Threshold for Impaired Fasting Glucose: Results from the Population-Based Omiya MA Cohort Study" by Masayuki Kato., which appeared in JAT 2009, 16: 857-861, are incorrect. The editiorial staff apologizes to the authors and all readres. The correct are presented with corresponding below.

Original Article

\section{Fasting Plasma Glucose and Incidence of Diabetes --- Implication for the Threshold for Impaired Fasting Glucose: Results from the Population-Based Omiya MA Cohort Study}

Masayuki Kato ${ }^{1}$, Mitsuhiko Noda ${ }^{2}$, Hiroshi Suga ${ }^{3}$, Masahiko Matsumoto ${ }^{3}$, and Yasunori Kanazawa ${ }^{4}$ for the Omiya MA Cohort Study Group

False

Correct

Results: The incidence of diabetes increased as the baseline FPG level increased and a similar pattern was observed irrespective of sex or age. The hazard ratios compared with subjects with FPG $<85 \mathrm{mg} / \mathrm{dL}$ adjusted for possible confounding factors were 3.83 (95\% confidence interval $(95 \% \mathrm{CI}) ; 2.41-6.08)$ for subjects with 100 to $104 \mathrm{mg} / \mathrm{dL}$ FPG and 7.97 (95\% CI; 4.98-12.4) for subjects with 105 to $109 \mathrm{mg} / \mathrm{dL}$ FPG.
Results: The incidence of diabetes increased as the baseline FPG level increased and a similar pattern was observed irrespective of sex or age. The hazard ratios compared with subjects with FPG $<85 \mathrm{mg} / \mathrm{dL}$ adjusted for possible confounding factors were 3.83 (95\% confidence interval $(95 \% \mathrm{CI}) ; 2.41-6.08)$ for subjects with 100 to $104 \mathrm{mg} / \mathrm{dL}$ FPG and 7.87 (95\% CI; 4.98-12.4) for subjects with 105 to $109 \mathrm{mg} / \mathrm{dL}$ FPG. 